\title{
Instantaneous quantum description of photonic wavefronts and applications
}

\author{
Andre Vatarescu \\ Fibre-Optic Transmission of Canberra, 32 Batman Street, Canberra, ACT 2612, Australia \\ andre_vatarescu@yahoo.com.au
}

\begin{abstract}
By imposing the condition of non-vanishing expectation values for the amplitude and phase of field operators, pure quantum states are identified composed of two consecutive number states. These pure states also deliver noise-free radiation modes restricting the "halfphoton noise" to the expectation value of the lowest level of dynamic and coherent number states. As a result, instantaneous phase-sensitive amplification of photons is easily controlled and direct evaluation of time or distance - varying wavefront distributions of photons and phases can be carried out for sub-Poissonian distributions of photons without the need for quasiprobabilities.
\end{abstract}

Key words: Quantum Rayleigh emission, optically linear parametric interactions, dynamic and coherent number states, sub-Poissonian photon distributions.

\section{Introduction}

Optical sources of sub-Poissonian radiation [1] would enhance the detection resolution of optical signals by virtue of reduced fluctuations in the number of photons and, possibly, better defined phases and states of polarization. A broad range of applications can be envisaged from optical sensing of environmental variables to improved signal-to-noise ratio in optical communications [2] provided controllable and highly-efficient sources could be developed.

It is commonly assumed that the sub-Poissonian distribution of photons is a quantum property of light because it requires a quasi-distribution for its description [1] in terms of coherent states of light (CSL). However, CSLs do not specify instantaneous properties of photonic wavefronts which are required for localized interactions between optical waves simultaneously propagating through the same dielectric medium. Yet, by identifying a physically meaningful quantum wavefunction [3], sub-Poissonian distributions can be generated with any number of photons for any overall distribution, by means of the optically linear parametric (OLP) amplification of photons in the "classical" regime [4-6].

In the context of parametric amplification of photons by a factor of $G$, for unsaturated gain, the expectation value $|\alpha|^{2}$ of the number of photons corresponding to a coherent state of light $|\alpha\rangle$ will become $G|\alpha|^{2}$, but, physically, the photon distribution will develop gaps between any two consecutive number states as $G n-G(n-1)=G$. Thus, the coherent state is destroyed and replaced, mathematically, by a mixed state of quasi-distributions [1] of coherent states. As a result, rather complicated and roundabout procedures are needed to, apparently, specify the number of photons in terms of quasi-probabilities of coherent states which are, themselves, expressed as a superposition of number states.

A criterion for generating sub-Poissonian distributions of photons through differential amplification of photons was outlined in [4] involving the phase-dependent gain coefficient. However, in order to design and control such sources, a physical model for amplification of any photon distribution and level of number states needs to be developed beyond the identification of the phase-dependent gain coefficient [4-7] accompanied by a parametric phase-pulling effect which shifts the phase of a weak signal towards a $(-\pi) / 2$ difference from that of strong pumps. Photon coupling processes involve the instantaneous values of the 
wavefronts interacting simultaneously at a point in space within a dielectric medium [4 -7].

A simple and practical photonic quantum state $\left|\Psi_{n}\right\rangle$ is needed which is capable of delivering the instantaneous number of photons of an optical wavefront, and the associated phase regardless of the overall distribution of the beam, so that $\left\langle\Psi_{\mathrm{n}}\right| \hat{a}^{\dagger}$ (or $\left.\hat{a}\right)\left|\Psi_{\mathrm{n}}\right\rangle \neq 0$ for the annihilation and creation operators, $\hat{a}$ and $\hat{a}^{\dagger}$, respectively. Such a state vector is readily identifiable in Section 2 below, from the two-component Hamiltonian of the optical field in free-space. The dynamic number states defined for a given number of photons as $\left|\Psi_{\mathrm{n}}(t)\right\rangle=$ $2^{-1 / 2}(|n\rangle+|n-1\rangle)$ can deliver a non-vanishing expectation value for the field operators. This will enable direct calculations of the wavefront number of photons and phase shifts stemming from interactions with dielectric media. This is in contrast to the quadrature eigenstates of $\left\langle x\left|\hat{a}^{\dagger}+\hat{a}\right| x\right\rangle \neq 0$ used in quantum tomography [8 - 9] which invoke, by analogy, the eigen-functions $|x\rangle$ of the coordinate, or position, operator of a mass particle harmonic oscillator. Nevertheless, the strength of the optical field is determined by the instantaneous number of photons [10] without any connection to the position of a harmonically moving mass particle. The spatial field profile of a group of photons is derived in the Appendix A below.

A long series of intensity measurements [8-9] of the instantaneous number of photons carried by the wavefronts reaching the detection surface will provide a probability distribution for the ensemble of the number states of the wavefronts forming the optical beam. Upon detection [8], a photonic wave front collapses into a particular number state whose values of number of photons, phase, wave vector, state of polarization, etc. would be stochastic. The sequence of optical wavefronts of the beam will form an ensemble of photonic systems composed of an ergodic distribution, i.e., within a long enough duration, the characteristics of the wavefronts are reproduced. These measured instantaneous values will build up probability distributions from which the ensemble expectation values and variances can be calculated. Nevertheless, it should be pointed out that the localized measurement method of quantum tomography [8 - 9] does not deal with photon couplings or interactions in a dielectric medium

Similarly, the definition of the phase eigenstates [11] leads to a rather problematic aspect as pointed out in [11]: "We note that a phase state is not a physical state because the expectation value of the photon number diverges as $s \rightarrow \infty$." [11, Sec. IV, p. 1668]

The optical field of a dynamic and coherent number state - derived in Section 2 from its equation of motion - will enable direct prediction and control of the output distribution of photons, for further signal processing and interactions, of both magnitudes and phases of the instantaneous wavefront values. Phase information will be obtained from the quadrature phase operators defined in Section 3. Physical properties of the dynamic and coherent states of light are discussed in Section 4 below. The advantages of the dynamic and coherent states in characterizing sub-Poissonian distributions of photons are outlined in Section 5 which is followed by brief conclusions. Appendix A below provides, for the first time, the spatial profile of the field of a group of photons associated with a wavefront.

\section{Optical fields of dynamic and coherent number states}

Photons and their instantaneous properties are detected and measured as a sequence of wavefront number states which make up a pure quantum state vector $|\Psi(\boldsymbol{r}, t)\rangle=$ $\sum_{n} c_{n}(\boldsymbol{r}, t)|\mathrm{n}\rangle$ regardless of the overall distribution to which the photons belong [8-9]. The quantum probability of occupation of an eigenstate is given by the normalized distribution - crossing a surface at location $\boldsymbol{r}$ - of the time-varying coefficients $\left|c_{n}\right|^{2}$ satisfying the condition $\sum_{n}\left|c_{n}\right|^{2}=1$, and orthogonality $\langle\mathrm{n} \mid \mathrm{m}\rangle=\delta_{\mathrm{n} m}$. The detection of photons occurs as a result of their optical field exchanging energy with electrons of the atomic structure of the detector, similarly to the Jaynes - Cummings model [12-13] for the quantized dipole-photon exchange of energy. The detection process collapses the photonic quantum state into an instantaneous 


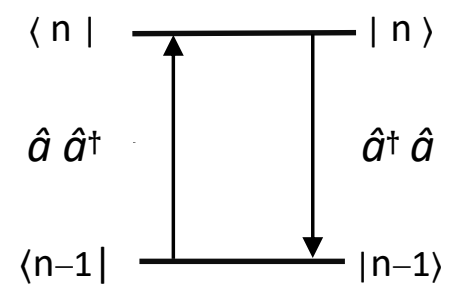

Fig. 1. An illustration of the dynamic and coherent two-component number states. We note that: $\langle\mathrm{n}| \hat{a}^{\dagger}=\sqrt{\mathrm{n}}\langle\mathrm{n}-1|$, and $\langle\mathrm{n}-1| \hat{a}=\sqrt{\mathrm{n}}\langle\mathrm{n}|$.

eigenvalue of a number operator [14] regardless of the ensemble distribution to which it belongs, e.g., a coherent state or an arbitrary distribution.

\subsection{Non-zero instantaneous optical field expectation values}

As the lowest detectable amount of optical energy $\hbar \omega$ corresponds to one photon, the presence of a half-photon per radiation mode in the form of vacuum noise fluctuations can only be physically meaningful as an expectation value of two consecutive number states, $[(n+1)+$ $\mathrm{n}] / 2=\mathrm{n}+0.5$; for $\mathrm{n}=0$ we obtain half a photon. Experiments conducted in a vacuum environment may still be affected by background radiation, e.g. of solar origin, or by black body radiation in line with Plank's formula.

In Appendix A below, the free-space Hamiltonian $\widehat{H}_{f}$ of an instantaneous photonic wavefront is readily derived, without invoking harmonic oscillators, in terms of a field operator $\hat{a}$ and its adjoint, $\hat{a}^{\dagger}$, to be:

$$
\begin{aligned}
& \widehat{H}_{f}=\hbar \cdot \omega \widehat{N}_{c} \\
& \widehat{N}_{c}=0.5\left(\hat{a}^{\dagger} \hat{a}+\hat{a} \hat{a}^{\dagger}\right)
\end{aligned}
$$

where $\widehat{N}_{c}$ is a complete number operator. Based on experimental results indicating that one photon is an indivisible amount of energy, we seek a wave function $\left|\Psi_{n}\right\rangle$ which will deliver the number of photons $n$ from the equalities:

$$
\begin{aligned}
& \left\langle\Psi_{\mathrm{n}}\left|\widehat{N}_{c}\right| \Psi_{\mathrm{n}}\right\rangle=n \\
& \left\langle\Psi_{\mathrm{n}}\left|\hat{a}^{\dagger} \hat{a}\right| \Psi_{\mathrm{n}}\right\rangle+\left\langle\Psi_{\mathrm{n}}\left|\hat{a} \hat{a}^{\dagger}\right| \Psi_{\mathrm{n}}\right\rangle=2 n
\end{aligned}
$$

so that, from Eqs. (1b) the number states $|\mathrm{n}\rangle$ are eigenfunctions, with different eigenvalues, of both of the product operator terms:

$$
\begin{aligned}
& \left\langle\mathrm{n}\left|\hat{a}^{\dagger} \cdot \hat{a}\right| \mathrm{n}\right\rangle=n \\
& \left\langle\mathrm{n}-1\left|\hat{a} \cdot \hat{a}^{\dagger}\right| \mathrm{n}-1\right\rangle=n ; \text { or }\left\langle\mathrm{n}\left|\hat{a} \cdot \hat{a}^{\dagger}\right| \mathrm{n}\right\rangle=n+1
\end{aligned}
$$

Thus, in the basis of number states, the commutator $\left[\hat{a}, \hat{a}^{\dagger}\right]=1$ is obtained as a result of the quantization rather than leading to it. This does not affect the choice of the superposition wave function $\left|\Psi_{\mathrm{n}}\right\rangle$ and related expectation values:

$$
\begin{aligned}
& \left|\Psi_{\mathrm{n}}\right\rangle=2^{-1 / 2}(|\mathrm{n}\rangle+|\mathrm{n}-1\rangle) \\
& \left\langle\Psi_{\mathrm{n}}\left|\hat{a}^{\dagger} \hat{a}\right| \Psi_{\mathrm{n}}\right\rangle=0.5(n+n-1)=n-1 / 2 \\
& \left\langle\Psi_{\mathrm{n}}\left|\hat{a} \hat{a}^{\dagger}\right| \Psi_{\mathrm{n}}\right\rangle=0.5(n+n+1)=n+1 / 2
\end{aligned}
$$




$$
\left\langle\Psi_{\mathrm{n}}\left|\widehat{N}_{c}\right| \Psi_{\mathrm{n}}\right\rangle=0.5 \times 2 n=n
$$

This relation satisfies the condition of Eq. (2a) and implies two opposite processes which can be interpreted as shifting a number state up or down by one photon which is the smallest indivisible amount of energy of an optical field. Their illustration can be seen in Fig.1. Experimentally, from the photoelectric effect, the optical fields consists of an integer number of the lowest indivisible amount of energy, i.e. photons. However, for the lowest state of $\mathrm{n}=1$, the expectation value is half a photon because $\left(\langle 0| \hat{a}^{\dagger}\right)(\hat{a}|0\rangle)=0$.

Analogously to the derivation [12] of coherent states $|\alpha\rangle$, the non-Hermiticity of the photon annihilation and creation operators allows for complex classical numbers ( $c$-numbers) to be delivered when these operators act on number states. Applying $\hat{a}$ and $\hat{a}^{\dagger}$ to $|\mathrm{n}\rangle$ returns a complex $c$ - number

$$
s_{n}=n^{1 / 2} \exp \left(-i \varphi_{n}\right)
$$

which will become the complex amplitude of the photonic state, so that: $\hat{a}|\mathrm{n}\rangle=s_{n}|\mathrm{n}-1\rangle$ and $\hat{a}^{\dagger}|\mathrm{n}-1\rangle=s *_{n}|\mathrm{n}\rangle$.

Equally, the annihilation and creation operators ( $\hat{a}$ and $\hat{a}^{\dagger}$, respectively) are adjoint operators of each other and interchange roles when acting on the Hermitian conjugate wave functions $\langle\mathrm{n}|$ of photon number states, i.e., $\langle\mathrm{n}-1| \hat{a}=s_{n}\langle\mathrm{n}|$ and $\langle\mathrm{n}| \hat{a}^{\dagger}=s^{*}{ }_{n}\langle\mathrm{n}-1|$. This feature enables the coherent state of light to generate a non-zero expectation value for the optical field.

We also impose the condition that the expectation values of the instantaneous complex field operators $\left\langle\Psi_{\mathrm{n}}|\hat{a}| \Psi_{\mathrm{n}}\right\rangle$ and $\left\langle\Psi_{\mathrm{n}}\left|\hat{a}^{\dagger}\right| \Psi_{\mathrm{n}}\right\rangle$ exhibit a sinusoidal time variation, which will be determined in the next sub-section from the Schrödinger equation of motion.

\subsection{Photonic wavefront interactions of dynamic and coherent number states}

The field operators $\hat{a}$ and its adjoint $\hat{a}^{\dagger}$ connect two consecutive number states, and consequently, a superposition of $|n-1\rangle$ and $|n\rangle$ should give rise to a non-zero optical field for the following state vector:

$\left|\Psi_{\mathrm{n}}(t)\right\rangle=c_{\mathrm{n}}(t)|\mathrm{n}\rangle+c_{\mathrm{n}-1}(t)|\mathrm{n}-1\rangle$

and the two probability amplitudes satisfy the equality $\left|c_{n}\right|^{2}+\left|c_{n-1}\right|^{2}=1$. The expansion coefficients $c_{j}(t)$ in Eq. (6) with $j=n$ and $n-1$, will change as a result of a transition between $|n-1\rangle$ and $|n\rangle$ as determined by the Schrödinger equation of motion

$i \hbar \frac{\partial}{\partial t}\left|\Psi_{n}(t)\right\rangle=\left(\hat{H}_{f}+\hat{H}_{i n t}\right)\left|\Psi_{n}(t)\right\rangle$

where an interaction Hamiltonian $\widehat{H}_{\text {int }}$ has been included for a later application. Since an operator remains unchanged in the Schrödinger picture, the rate of change for the expectation value in the Schrödinger picture contains only a commutative term between the operator and the Hamiltonian:

$i \hbar \frac{\partial}{\partial t}\left\langle\Psi_{n}(t)|\hat{a}| \Psi_{n}\right\rangle=\left\langle\Psi_{n}\left|\left[\hat{a}, \hat{H}_{f}\right]\right| \Psi_{n}\right\rangle$

The commutative relations involving the field Hamiltonian are:

$\left[\hat{a}, \widehat{H}_{f}\right]=\hbar \omega \hat{a} ;$ and $\left[\hat{a}^{\dagger}, \widehat{H}_{f}\right]=-\hbar \omega \hat{a}^{\dagger}$

The expectation value of the quadrature operator $\left(\hat{a}+\hat{a}^{\dagger}\right)$ for the wave function of Eq. (6), 
is given, at time $t$, by

$\left\langle\Psi_{\mathrm{n}}(t)\left|\left(\hat{a}+\hat{a}^{\dagger}\right)\right| \Psi_{\mathrm{n}}(t)\right\rangle=\mathrm{c}_{\mathrm{n}}(t) \mathrm{c}^{*}{ }_{\mathrm{n}-1}(t) s_{\mathrm{n}}+\mathrm{c}^{*}{ }_{\mathrm{n}}(t) \mathrm{c}_{\mathrm{n}-1}(t) s^{*}{ }_{\mathrm{n}}$

We note that the last equality contains two complex conjugate terms and define: $\eta(t)=\mathrm{c}_{\mathrm{n}}(t) \mathrm{c}^{*}{ }_{\mathrm{n}-1}(t)$

to obtain from Eq. (10)

$$
\left\langle\Psi_{\mathrm{n}}(t)|\hat{a}| \Psi_{\mathrm{n}}(t)\right\rangle=\eta(t) s_{\mathrm{n}}
$$

The solution for $\eta(t)$ is found from the equation of motion derived by combining Eqs. (8-9) and (11-12) leading to:

$$
\begin{aligned}
& \frac{\partial}{\partial t} \eta(t)=-i \omega \eta(t) \\
& \eta(t)=\eta(0) e^{-i(\omega t+\varphi(0))}
\end{aligned}
$$

With $\left|\mathrm{c}_{\mathrm{n}}(t)\right|^{2}+\left|\mathrm{c}_{\mathrm{n}-1}(t)\right|^{2}=1$, one estimates the peak value of $\eta(0)=0.5$, and Eq. (12) becomes:

$$
\left\langle\Psi_{\mathrm{n}}(t)|\hat{a}| \Psi_{\mathrm{n}}(t)\right\rangle=0.5 e^{-i(\omega t+\varphi(0))} n^{1 / 2} e^{-i \varphi \mathrm{n}}
$$

delivering, along with its complex conjugate from Eq. (10) above, an instantaneous expectation value for an optical field of any level of photon number, and associated phase regardless of the overall beam distribution, and corresponding to a classical field representation. The quadrature operators and their expectations values are identified in Section 3 below, while interference patterns of two such photonic wavefronts are presented in Section 4 below.

The conventional expectation number of photons for this dynamic state of Eq. (6) is found to be:

$$
\left\langle\Psi_{\mathrm{n}}(t)\left|\hat{a}^{\dagger} \hat{a}\right| \Psi_{\mathrm{n}}(t)\right\rangle=\mathrm{n}-\left|\mathrm{c}_{2}(t)\right|^{2}
$$

The factor $\left|c_{2}(t)\right|^{2}=1 / 2$ balances the half-photon noise.

Having identified a quantum wave function capable of delivering the instantaneous magnitude and phase of an optical field, we can now apply the formalism of [4-5] to the propagation along an optical waveguide directional coupler by employing the following composite photonic quantum state function $|\Phi\rangle$ for two optical waves identified by their waveguide, their Hamiltonian of interaction and the equation of motion:

$$
\begin{aligned}
& |\Phi\rangle=\left|\Psi_{\mathrm{n}, 1}\right\rangle\left|\Psi_{\mathrm{n}, 2}\right\rangle \\
& \hat{H}_{i n t}=\hbar \omega \chi^{(1)}\left(\hat{a}_{2}^{\dagger} \hat{a}_{1}+\hat{a}_{2} \hat{a}_{1}^{\dagger}\right) \\
& \frac{\partial}{\partial t}\left\langle\Phi\left|\hat{a}_{1}\right| \Phi\right\rangle=-i \omega \chi^{(1)}\left\langle\Phi\left|\hat{a}_{2}\right| \Phi\right\rangle
\end{aligned}
$$

where $\chi^{(1)}$ is the real part of the first-order susceptibility of the dielectric medium. This interaction will modify the complex field amplitude $s_{\mathrm{n},, j}(j=1,2)$ of two co-propagating and overlapping optical beams of the same frequency, identifiable by their respective optical waveguides $(j=1,2)$. From Eq. (15) we have for the expectation value of the optical field

$$
\left\langle\Phi\left|\hat{a}_{j}\right| \Phi\right\rangle=0.5 e^{-i\left(\omega t+\varphi_{j}(0)\right)} s_{\mathrm{n}, j}
$$


After converting to number of photons, i.e., $\left|s_{\mathrm{n}, j}\right|^{2}=N_{j}$ and corresponding phases $\varphi_{j}$, the equation of motion (19) provides the rates of change of $N_{j}$ and $\varphi_{j}$ as follows [4 -6]:

$$
\begin{aligned}
& \frac{\partial}{\partial t} N_{1}=g_{1} N_{1} \\
& g_{1}=-2 \kappa r_{21} \sin \theta_{21} \\
& \frac{\partial}{\partial t} \varphi_{1}=\kappa r_{21} \cos \theta_{21} \\
& r_{2 I}=\left(N_{2} / N_{1}\right)^{1 / 2} \\
& \kappa=\frac{k_{o}}{2 n} \iint d x d y \chi^{(1)} f_{1} f_{2} \boldsymbol{e}_{1} \cdot \boldsymbol{e}_{2}
\end{aligned}
$$

where the gain coefficient $g$ includes an overall coupling coefficient $\kappa$ defined in [6] which depends on the polarization states of the photons $\boldsymbol{e}_{1}$ and $\boldsymbol{e}_{2}$. The phase difference between the two waves is $\theta_{21}=\left(\beta_{2}-\beta_{1}\right) z+\varphi_{2}-\varphi_{1}, \beta$ being the propagation constant and $z / t=\mathrm{v}_{\mathrm{p}}$ is the phase velocity. In Eq. (21e), $k_{\mathrm{o}}$ and $n$ specify the free-space wavevector and the effective refractive index, respectively. It should be noted that Eqs. (21) describe the physically meaningful process of quantum Rayleigh conversion of photons [4 - 6]. The coupling coefficient of Eq. (21e) indicates that the entire local value of the optically linear susceptibility $\chi^{(1)}$ is involved in the coupling process in the dielectric medium at any point where the two spatial distributions $f_{1}$ and $f_{2}$ overlap, each having units of $\mathrm{m}^{-1}$, and the squares $f^{2}$ are normalized to a dimensionless unit over the cross-section area. This is in contrast to the physically impossible coupling between two optical waveguides apparently induced by a perturbation of the dielectric constant in the cladding - see reference [6] for details.

It is noteworthy that the phase of the dynamic and coherent number state can be derived from both the $c$ - number of the field operator of Eq. (5) and from the equation of motion of the expectation value of the field operator in Eqs. (13-14). Properties of these phases are investigated in the following Section.

\section{Coherence of dynamic number states}

The Heisenberg uncertainty principle [14] has to do with the joint statistical distribution of the measured values of two variables corresponding to repeated measurements of identically prepared systems [8-9], and applies to the simultaneous measurements of two dynamic variables whose quantum operators do not commute in a given set of state wave functions, the variables being incompatible observables.

The principle of uncertainty does not preclude the existence of well-defined numerical values for either variable [14]. Limited information about one variable is associated with a variance in the measured values with more information being available about the other variable. Measurements yield precise instantaneous values - within the constraints of the equipment by collapsing the system's wave function into a specific value [14]. A quantum "spread" implies that measurements on identically prepared systems do not return identical results because of system-related fluctuations such as spontaneous emission, time-varying losses, temperature variations, etc.

One should not confuse prediction with measurements. It is the measured values that come into play when plotting the joint distribution of two observable physical variables. A measurement of only one variable will still have its own variance of values but it is not subject to the Heisenberg uncertainty principle, because the physical system is not disturbed by the measurement of another variable. One can easily measure a well-defined eigenvalue of an 
operator [14] at a given time.

In free space, the degree of coherence of an optical monochromatic wave would be characterized by the statistical phase variance of the ergodic evolution of the wavefront phases. Their measured statistical distribution - if detected in conjunction with another observable physical quantity or variable - will be subject to the Heisenberg uncertainty principle characterizing statistical measurements. However, each instantaneous wave front has, possibly, a time-varying, and - as a result of interacting with the dielectric medium which collapses the wave function - a well-defined phase which will be involved in the optically linear parametric interactions in a dielectric medium [4 -7].

Two dynamic number states - derived in the previous Section 2 - co-propagating through a dielectric medium will couple photons from one state to the other depending on the relative phase between the two waves. This process, repeatedly, will eliminate optical waves whose phases diverge substantially from the phase of the surviving wave which will dominate the output of a lasing cavity.

Quadrature phase operators [15] $\hat{C}$ and $\hat{S}$ defined in terms of the field operators and the conventional number operator $\hat{N}=\hat{a}^{\dagger} \hat{a}$, have the form for $\mathrm{n}>0$ :

$\hat{C}=\hat{a} \hat{N}^{-1 / 2}+\hat{N}^{-1 / 2} \hat{a}^{+}$

$\hat{S}=i\left(\hat{a} \hat{N}^{-1 / 2}-\hat{N}^{-1 / 2} \hat{a}^{\dagger}\right)$

and will provide information about the phase associated with the dynamic number states. With the photon number operator $\hat{N}$ returning the number of photons in the basis of number states i.e., $\hat{N}|\mathrm{n}\rangle=\mathrm{n}|\mathrm{n}\rangle$, any operator function $f(\hat{N})$ can be expanded in a power series and replaced in terms of the number of photons, as follows [12]:

$f(\hat{N})=\sum_{p} d_{p} \hat{N}^{\mathbf{P}} ; f(\hat{N})|\mathrm{n}\rangle=f(\mathrm{n})|\mathrm{n}\rangle ; \hat{N}^{-1 / 2}|\mathrm{n}\rangle=\mathrm{n}^{-1 / 2}|\mathrm{n}\rangle$

where $d_{p}$ are expansion coefficients. For the particular case of the zero photon state $|0\rangle$, the product operator needs to be inverted [15] by substituting $\hat{a} \hat{a}^{\dagger}=\hat{a}^{\dagger} \hat{a}+1$ into the power series expansion of $\hat{a} f(\hat{N})$ to obtain $f(\hat{N}+1) \hat{a}$.

From Eqs. (15), (20) and (22) we find the following expectation values for the phase quadratures:

$$
\begin{aligned}
& \left\langle\Phi\left|\hat{C}_{j}\right| \Phi\right\rangle=\cos \left(\omega t+\varphi_{j}\right) \\
& \left\langle\Phi\left|\hat{S}_{j}\right| \Phi\right\rangle=\sin \left(\omega t+\varphi_{j}\right)
\end{aligned}
$$

These variables will be measured by the method of balanced homodyne detection [12]. The dynamic and coherent number states (DCNS) identified in Eq. (6) possess an optical field which can be compared with the classical field. Consequences of these properties are discussed in the following Section.

\section{Physical aspects of dynamic and coherent number states}

In so far as localized detection of photons of two dynamic and coherent number states is concerned, the photocurrent $I_{p h}$ generated by the interference output of a balanced homodyne detector is calculated by combining equations (17), (20) and (24) to obtain:

$$
\begin{aligned}
I_{p h}(t) & =K\left\langle\Phi\left|\left(\begin{array}{llll}
\hat{a}_{1}^{\dagger} & \hat{a}_{2}+\hat{a}_{1} & \hat{a}_{2}^{\dagger}
\end{array}\right)\right| \Phi\right\rangle= \\
& =2 K\left(\begin{array}{lll}
N_{1} & N_{2}
\end{array}\right)^{1 / 2} \cos \left(\varphi_{1}-\varphi_{2}\right)
\end{aligned}
$$

with the constant of proportionality $K$ corresponding to the quantum efficiency of photon- 
to - electron conversion.

The statistical properties of a stochastic and ergodic optical beam will unfold in time upon detection at a point in space. Intensity measurements absorb photons leaving nothing for phasemeasurements. Splitting a beam in two and measuring quadrature phase values of $\cos \varphi$ and $\sin \varphi$ will leave nothing for intensity measurements. A stable reference beam is needed for all types of measurements. Its fluctuations, in fact, may be mistaken for proof of the uncertainty principle.

Two number states $|n\rangle$ and $|m\rangle$ propagating simultaneously across the same dielectric medium would exchange photons, parametrically, with each other through the real part of the susceptibility, see Eqs. (21) above, with the possibility of creating the $|n+m\rangle$ number state.

A criterion for generating sub-Poissonian distributions of photons through differential amplification of photons was outlined in [4] involving the phase-dependent gain coefficient, and the following Section presents a straightforward description of such photon distributions.

\section{Sub-Poissonian distribution of photons}

With the evolution of an optical wavefront being described by Eqs. (21), the number state is identified by the number of photons emerging from a parametric interaction, i.e., $\mathrm{n}=N_{1}$, so that the overall statistical distribution of the beam is given by the elements $\rho_{n n}$ of the density matrix of the mixed state.

For the "input" and "output" corresponding to one photon interaction, the probability coefficients take on the values:

One lost photon: input $\quad c_{n}=1 ; c_{n-1}=0 ;$

output $c_{n}=0 ; c_{n-1}=1 ;$

One gained photon: input $\quad c_{n}=0 ; c_{n-1}=1$;

output $\quad c_{n}=1 ; c_{n-1}=0 ;$

so that, the orthogonality of the number states can be used to specify clearly the pure state and mixed states of light, which is not the case with the coherent states of light which lack orthogonality.

The photon number variance in the basis of number states is calculated from:

$\left\langle(\Delta \mathrm{n})^{2}\right\rangle=\left\langle\hat{N}^{2}\right\rangle-\langle\hat{N}\rangle^{2}=\operatorname{Tr}\left(\hat{\rho} \hat{N}^{2}\right)-[\operatorname{Tr}(\hat{\rho} \hat{N})]^{2}=\sum_{\mathrm{n}} \rho_{\mathrm{n} n} \mathrm{n}^{2}-\left(\sum_{\mathrm{n}} \rho_{\mathrm{n} n \mathrm{n}}\right)^{2}$

For a pure number state of the wavefront $\rho_{n} n=\delta_{n m}$ leading to the well-known result of $\rho_{\mathrm{m} \mathrm{m}}=1$ and $\left\langle(\Delta \mathrm{n})^{2}\right\rangle=0$. For the mixed state of the beam $\rho_{\mathrm{n} n}<1$ and the variance broadens as more states are occupied. For a sub-Poissonian distribution, the reference Poissonian state will have the same mean number of photons, and the variance difference is given by

$$
\left\langle(\Delta n)^{2}\right\rangle-\langle n\rangle=\sum_{n}\left(\rho_{n n}-\rho_{n n}^{(P)}\right) n^{2}
$$

where $\rho^{(\mathrm{P})}{ }_{\mathrm{n} n}$ is the Poissonian distribution for which $\left\langle(\Delta \mathrm{n})^{2}\right\rangle^{(\mathrm{P})}=\langle\mathrm{n}\rangle$. In this way, the spread is determined by the difference between the density matrix elements, and no quasiprobabilities are needed as in ref. [1].

This relative relation of Eq. (27) stems from the fact that the number states are eigenfunctions of the photon number operator, which is not the case for the coherent states of light as they are destroyed by the creation operator. This shortcoming of the coherent states of light was handled by re-arranging the order of the field operators leading to three types of quasiprobabilities on the complex phase-space of the eigenvalues $\alpha$ [1].

Additionally, deficiencies of the formalism based on the Bogoliubov solution for the parametric amplification are detailed in [4-5]. This physically impossible solution for the optical field operators was used in the Wigner quasi-probability (WQP) [11-12] to distort the 
circular distribution of a coherent state into an elliptic distribution - on the phase-space of eigenvalues $\alpha$ of the coherent states - for noise squeezing. But, the WQP itself distorts the point-like distribution of a pure coherent state in the context of the $P$ quasi-probability. By contrast, a physically meaningful process of photonic coupling between two quadratures of an optical field was identified in [6, Eq. (5)], brought about by the quantum Rayleigh stimulated emission amplifying one quadrature while attenuating the other one.

\section{Conclusions}

Based on the physical process of photon detection triggered by the optical field, quantum two-component number states are identified. These states deliver through their equation of motion a non-vanishing expectation value for the field operators.

The possibility of evaluating directly, without the need for quasi-probabilities, the evolution of an optical wavefront regardless of the photon statistics of the overall beam, will facilitate the design and operation of functional integrated photonic circuits and devices.

\section{Appendix A-The spatial field profile of photons}

The following analysis provides a physically meaningful description of the optical field carried by a photonic wavefront of any number of photons and possessing an arbitrary phase.

Based on the formalism presented in [6], [16], the magnitude of the Poynting vector, i.e. the flux of energy $\mathcal{E}$ ( or number of photons ) carried by an optical wavefront of frequency $\omega$ and crossing a plane surface at position $z$ is given in terms of the electromagnetic field magnitudes $E$ and $B$, or corresponding operators, by the equalities

$\mathcal{E}=\omega \varepsilon E^{2}+c^{2} \omega B^{2}=0.5 \hbar \cdot \omega\left(a a^{*}+a^{*} a\right)$

with $a=(\varepsilon / \hbar)^{1 / 2}(E+i c B)$ and its complex conjugate $a^{*}$.

From this relation one defines the annihilation and creation operators [10], [16] as:

$\hat{a}=(\varepsilon / \hbar)^{1 / 2}(\hat{E}+i c \hat{B})$ and $\hat{a}^{\dagger}=(\varepsilon / \hbar)^{1 / 2}(\hat{E}-i c \hat{B})$

with $\varepsilon$ and $\hbar$ indicating the permittivity of the medium and the reduced Planck constant, respectively.

By using the wave function $\left|\Psi_{n}\right\rangle$ from Eq. (15) two equations can be identified for the expectation values of $\hat{a}$, or the corresponding $c$ - numbers, which are:

$$
\begin{aligned}
& \left\langle\Psi_{\mathrm{n}}|\hat{a}| \Psi_{\mathrm{n}}\right\rangle=b\langle\hat{E}\rangle+i s\langle\hat{B}\rangle \\
& \left\langle\Psi_{\mathrm{n}}|\hat{a}| \Psi_{\mathrm{n}}\right\rangle=q e^{-i \omega t}
\end{aligned}
$$

where $q=0.5 \sqrt{ } \mathrm{n}, b=(\varepsilon / \hbar)^{1 / 2}$ and, $s=(\varepsilon c / \hbar)^{1 / 2}$. We point out that both quadratures of the field are represented in the phasor notation of Eqs. (A3 - A4). Eq. (A3) is obtained from Eq. (A2) and can be expressed in terms of the $c$-numbers $E=\langle\hat{E}\rangle$ and $B=\langle\hat{B}\rangle$. Recalling the relations [16] between the vector potential $\boldsymbol{A}(z, t)$ and the fields as

$$
\boldsymbol{E}=-\partial \boldsymbol{A} / \partial t \quad \text { and } \quad \boldsymbol{B}=\nabla \times \boldsymbol{A}
$$

in the Cartesian frame of coordinates $(x, y, z)$, the vectors have the notation, in the plane wave approximation: $\boldsymbol{A}=(A, 0,0) ; \quad \boldsymbol{E}=(E, 0,0) ; \boldsymbol{B}=(0, B, 0)$ and the wave vector $\boldsymbol{k}=\left(i k_{x}, i k_{y}, \beta\right)$ for a beam propagating in the $z$-direction in an optical waveguide. The complex amplitude of the vector potential is represented by

$$
A(z, t)=A_{p}(z) f(x, y) e^{-i(\omega t-\beta z)}
$$

where the lateral profile of the guided mode is given by $f(x, y)$ and the propagation constant by $\beta=$ $2 \pi n_{\text {eff }} / \lambda$. The second term of the curl operation $\nabla \mathbf{x} f(x, y) \mathbf{x}=(\partial f / \partial z) \mathbf{y}-(\partial f / \partial y) \mathbf{z}$ does 
not lead to wave propagation and does not affect measurements in a plane perpendicular to the $z$ coordinate. The second term will therefore be set aside in the remainder of this analytic derivation.

Relating $A_{p}$ to a moving source of photons would suggest a relative distance $\zeta=z-z_{\mathrm{o}}$ with $z_{\mathrm{o}}$ being the temporal location of the photons and the localization given by a Dirac delta function $\delta\left(z-z_{0}\right)$, resulting in this differential equation after substituting Eqs. (A5- A6) into Eqs. (A3-A4) multiplied by the propagation phase $\exp i(\beta z)$ of the annihilation operator [4]:

$$
\frac{\partial A_{p}}{\partial z}+\sigma A_{p}=\gamma \delta\left(z-z_{\mathrm{o}}\right)
$$

where $\sigma=b \omega / s+i \beta=(\omega / c)\left(1+i n_{\text {eff }}\right)$, and $\gamma=-i q / s=i 0.5(n \hbar / \varepsilon)^{1 / 2}$. Setting $A_{p}(z)=g(z) e^{-\sigma \mathrm{z}}$

and inserting into the differential equation (A7) leads to:

$$
\int d g=\gamma \int e^{\sigma \mathrm{z}} \delta\left(z-z_{\mathrm{o}}\right) d z \quad \text { and } \quad g=\gamma e^{\sigma \mathrm{z}_{\mathrm{o}}}
$$

and for reasons of physical symmetry, after combining Eqs. (A8) and (A9), the spatial distribution of the magnitude of the vector potential generated by photons is found to be:

$A_{p}(z)=\gamma e^{-\sigma\left|z-\mathrm{z}_{\mathrm{o}}\right|}$

with $z_{\mathrm{o}}$ being the temporary location of the wavefront photons, and the vector potential's decay constant is inversely proportional to the wavelength $\lambda$ through $\operatorname{Re} \sigma=2 \pi / \lambda$. Thus, the local optical field includes contributions from photons in the vicinity of $z_{\mathrm{o}}$.

\section{References}

1. L. Davidovich, "Sub-Poissonian processes in quantum optics," Rev. Mod. Phys., vol. 68, no. 1, pp. 127-173, January 1996.

2. K. Kikuchi,"“Fundamentals of Coherent Optical Fiber Communications,” J. Lightwave Technol., vol. 34, no. 1, pp. 157-178, January 2016.

3. A. Vatarescu, Instantaneous Quantum Description of Photonic Wavefronts for Phase-Sensitive Amplification, Frontiers in Optics/Laser Science Conference (FiO/LS), paper JW4A.109, Washington, Sept. 2018.

4. A. Vatarescu, "Photonic Quantum Noise Reduction with Low-Pump Parametric Amplifiers for Photonic Integrated Circuits", Photonics, vol. 3, article 61, 2016.

5. A. Vatarescu A, "Phase-Sensitive Amplification with Low Pump Power for Integrated Photonics, "OSA Advanced Photonics Congress, paper ID: IM3A.6., 2016

6. A. Vatarescu, "Photonic coupling between quadrature states of light in a homogeneous and optically linear dielectric medium," J. Opt. Soc. Am. B, vol. 31, no. 7, pp. 1741-1745, July 2014.

7. A. Vatarescu "Photonic quadrature-wave pulses generated by a single electro-optic waveguide modulator for digital transmission," J. Opt. Soc. Am. B, vol. 32, no. 4, pp. 555-561, April 2015.

8. G. Breitenbach, S. Schiller, and J. Mlynek, "Measurement of the quantum states of squeezed light," Nature, vol. 387, pp. 471-475, 29 May 1997.

9. A. I. Lvovsky and M. G. Raymer, "Continuous-variable optical quantum-state tomography," Rev. Mod. Phys., vol. 81, no. 1, pp. 299-332, 2009.

10. R. J. Glauber and M. Lewenstein, "Quantum optics of dielectric media," Phys. Rev. A, vol. 43, no. 1, pp. 467491, January 1991.

11. D. T. Pegg and S. M. Barnett, "Phase properties of the quantized single-mode electromagnetic field," Phys. Rev. A, vol. 39, no. 4, pp. $1665-1675,1989$.

12. J. C. Garrison and R.Y. Chiao, Quantum Optics, Oxford University Press, 2008.

13. D. A. Steck, Quantum and Atom Optics, University of Oregon, available online at http://steck.us/teaching (revision 0.11.0, 18 August 2016).

14. D. J. Griffiths, Introduction to Quantum Mechanics, Publisher: Pearson Prentice Hall, 2005.

15. P. Carruthers and M. M. Nieto, "Phase and Angle Variables in Quantum Mechanics," Rev. Mod. Phys., vol. 40, no. 2, pp. 411-440, 1 April 1968.

16. K. J. Blow, R. Loudon, S. J. D. Phoenix and T. J. Shepherd, “Continuum fields in quantum optics," Phys. Rev. A, vol. 42, no. 7, pp. 4102- 4114, 1 October 1990. 